\title{
A Short Biography of Chia-Chiao Lin
}

\section{by Zuoqiang Shi}

\author{
Department of Mathematical Sciences, Tsinghua University
}

Chia-Chiao Lin (林家趐, July 7, 1916 to January 13, 2013), a native of Fuchou in the Fujian province, was born in Beijing. His father Kai Lin worked for the Ministry of Railways during the latter years of the Qing Dynasty. Also noteworthy is the interesting fact that his uncle Xu Lin (林 旭) was one of the so-called “Six Gentlemen” (六君子) who sacrificed their lives during the well-known Hundred Days' Reform in 1898. In 1934, Lin was admitted to the Department of Physics of Tsinghua University, achieving the highest score on the entrance exam. He graduated in 1937 as the student ranked highest amongst his entire graduating class. This was also a time of great turmoil, as it marked the year that Japan invaded China. Tsinghua University was relocated to the southwestern city of Kunming, and was joined with both Peking University and Nankai University, forming what would be called the Western-Southern United University. Lin was offered a teaching assistantship position in the Department of Physics at this newly created university.

Then, in 1939, he was awarded a scholarship, the Boxer Rebellion Indemnity Scholarship, from the China Education and Culture Foundation in order to help him pursue his graduate studies in the United Kingdom. Due to the sudden breakout of World War II in Europe, however, Lin's (as well as several other students') journey to Europe came to an abrupt halt in Japan, where he was forced to return to Kunming. With the aid of his mentor, Professor Pei-Yuan Zhou (周培源), he was later reassigned to University of Toronto in Canada. It was in 1940 that Lin arrived in Canada via India, and there he studied mathematical physics under J. L. Synge. In 1941, Lin earned his M.Sc. Degree and transferred to the California Institute of Technology in order to continue his studies under the supervision of Theodore von Kármán.

During this period, Lin solved an outstanding problem concerning the stability of parallel flows. Throughout nature, many of the flows we encounter are turbulent. Consequently, understanding the transition process from a laminar flow to a turbulent flow constitutes one of the major concerns facing scientists, and instability is considered to be one of the most likely mechanisms facilitating this transition. In mathematical terms, this instability corresponds to the eigenvalue problem for the Orr-Sommerfeld equation. Lin not only provided a beau- tiful mathematical solution to this eigenvalue problem, but also demonstrated that the viscosity of the fluid may, surprisingly, destabilize the flow! This seems to contradict our physical intuition, which suggests that the viscosity should actually stabilize the flow. Lin's work thus revealed the dual nature inherent in the stabilizing effects of viscosity. In order to illustrate this duality, we will consider, as an example, a parallel flow. For inviscid parallel flow, we know that the flow is stable; however, if we include the effects of viscosity, this parallel flow may, in fact, become unstable-a result that was later confirmed by experiments. With this result furnishing a thesis, Lin obtained his $\mathrm{PhD}$ in 1944, after which he taught at Caltech from 1943 to 1945 . From here, in light of his outstanding accomplishments, the Department of Applied Mathematics at the Brown University offered Lin a junior faculty position in 1945. Two years later, he was appointed as an Associate Professor in the Department of Applied Mathematics at the Massachusetts Institute of Technology. After joining the MIT faculty in 1947, he remained as a Professor of Mathematics, and later an Institute Professor, until he retired in 1987.

Another exceptional contribution made by Lin was the creation of his density wave theory, which allows us to explain galactic spiral structure. In 1959, he was invited by the Nobel Laureate Chen-Ning Yang (楊振寧) to visit the Institute for Advanced Study at Princeton for one year. During this period, he met the famous astronomer Bengt Strömgren. Moreover, during his visit to IAS, Strömgren invited Lin to attend an astronomical conference, where Lin learned about a problem concerning galactic differential rotation and the famous Winding Dilemma. The Winding Dilemma can be stated roughly as follows: Stars closer to the center of a spiral galaxy do not need to travel as far as those further from the center in order to complete an orbit; thus, inner stars should orbit more frequently than outer stars. The result of such motion would be a spiral that gradually winds up as the galaxy ages. Observations, however, of spiral galaxies at various distances and thus at different stages in their evolution have shown that this is not the case. This puzzling paradox is called the Winding Dilemma, and it has perplexed astronomers for many years. Lin's insight was realizing that the arms are definitely not composed of the same mat- 
ter-instead, they appear to be composed of density waves. Furthermore, he found that spiral density waves can be derived from the fluid equations and he discovered an approximate solution. In the following few years, he established the well-known spiral density wave theory. This theory successfully explains the main features of the spiral structure of the disk galaxies, thereby confirming that the observed spiral arms are density waves, rather than material arms, and this provided a beautiful solution to the Winding Dilemma.

Due to these distinguished achievements, as well as others, in the field of applied mathematics, Lin was elected as a member of the National Academy of Arts and Sciences in 1951, a member of Academia Sinica (Taipei) in 1958, and a member of the National Academy of Sciences in 1962. In 1987, he was awarded an honorary doctorate, as well as an honorary professorship, by Tsinghua University, and became a foreign member of the Chinese Academy of Sciences (Beijing) in 1994. Lin has also served as the Chairman of the Committee of Applied Mathematics of American Mathematical Society and the President of the Society of Industrial and Applied Mathematics (SIAM). Other honors and recognitions include the Timoshenko Medal from the American Society of Mechanical Engineers, the NAS Award in Applied Mathematics and Numerical Analysis, and the Fluid Dynamics Prize from the American Physical Society.

Chia-Chiao Lin has always shown great interest and concern in the development of the science and technology in China. Since 1972 he has visited China numerous times, in addition to inviting multiple well-known experts to give lectures in China. Furthermore, Lin arranged for many Chinese scholars to study and conduct research at MIT. Many of these scholars have gone on to become leaders in various fields in China. In November 2001, Lin accepted the appointment as a distinguished professor of Tsinghua University and in the following year moved back to Tsinghua University permanently. He also established the Pei-Yuan Zhou Center for Applied Mathematics at Tsinghua University in 2002, for which he served as the honorary director.

In 2013 Lin died of heart failure in Beijing. 